\title{
Incidence and Determinants of Transfusion- Transmissible Infections in Voluntary Blood Donors in Malawi, 2005-2015
}

Constance Wose Kinge ( $\nabla$ conskinge@yahoo.ca )

University of the Witwatersrand https://orcid.org/0000-0002-6221-5459

Juliana Kagura

University of the Witwatersrand Faculty of Health Sciences

Bridon M'baya

Malawi Blood Transfusion Services

Stephen Njolomole

Malawi Blood Transfusion Services

Charles Chasela

University of the Witwatersrand Faculty of Health Sciences

Research article

Keywords: TTI, HIV, HBV, HCV, blood donors

Posted Date: December 3rd, 2019

DOl: https://doi.org/10.21203/rs.2.18048/v1

License: (9) This work is licensed under a Creative Commons Attribution 4.0 International License.

Read Full License 


\section{Abstract}

Background Blood transfusion has been associated with a high risk of transfusion-transmissible infections (TTIs). These infections pose great threats to the availability and safety of blood supply for transfusion, particularly in sub-Saharan Africa (SSA) where the burden of disease is alarmingly high. We describe the incidence and determinants of TTls to help target interventions for safety and increased access to safe blood.

Methods This was secondary data analysis of a cohort of blood donors from the Malawi Blood Transfusion Service (MBTS) who donated blood from 2005-2015. Incidence was obtained by dividing the number of new cases by the total person-years at risk and survival probabilities computed by KaplanMeier estimates. Logistic regressions were used for risk factors.

Results We analysed data from 47,075 registered blood donors of which the majority were male ( $84 \%)$ with a median age of 22 years (IQR=18-22). Of the registered donors, 3,439 $(7.31 \%)$ were infected with at least one TTI (HIV, HCV or HBV). HBV was the most common TTI with $2.63 \%(n=1,238)$, followed by HIV with $1.74 \%(n=818)$ and HCV with $1.28 \%(n=602)$. Overall, TTI incidence was 43.4 per 10,000. Donors aged $20-24(\mathrm{OR}=2.15,95 \% \mathrm{Cl}=1.35-3.40)$, and $30-34(\mathrm{OR}=2.68,95 \% \mathrm{Cl}=1.67-4.32)$, males $(\mathrm{OR}=1.65$; $95 \% \mathrm{Cl}=1.47-1.85)$, and married donors $(\mathrm{OR}=1.93 ; 95 \% \mathrm{Cl}=1.38-2.69)$ had significantly higher odds of $\mathrm{TTI}$ in the multivariate logistic model. Infection with syphilis was a common significant risk factor for incident $\mathrm{HIV}(\mathrm{OR}=2.62,95 \% \mathrm{Cl}=1.57-4.38), \mathrm{HCV}(\mathrm{OR}=2.03,95 \% \mathrm{Cl}=1.04-3.98)$, and $\mathrm{HBV}(\mathrm{OR}=1.71$, $95 \% \mathrm{Cl}=1.01-2.89)$.

Conclusion The overall incidence of TTIs in the Malawian donor population is comparatively low. The incidence of HIV, HCV and HBV is high in males, the unemployed, donors living in the Central Region, involved in high-risk sexual behaviour, and co-infected with syphilis. HBV is most common among supposedly healthy donors, followed by HIV and HCV. TTI therefore, remains a cause for concern toward availability and safe blood supply. Implementation of strict and proper donor selection criteria and continuous screening for TTI indicators can help maximise safe blood supply and transfusion.

\section{Introduction}

Providing safe and sufficient blood and blood product supplies is of key public health importance. This is especially so in sub-Saharan Africa where there is a high burden of transfusion-transmissible Infections (TTIs) such as the human immunodeficiency virus (HIV), hepatitis B virus (HBV), hepatitis C virus (HCV), malaria and syphilis amongst others. The worldwide estimates by the World Health Organization (WHO) of people living with HIV, chronic HBV and HCV sits at 37.9, 257 and 71 million, respectively $(1,2)$.

Safe and reliable transfusion services remain largely unavailable to the world's poorest populations, particularly in sub-Saharan Africa (3). Despite high demands for blood supplies, Malawi faces a serious problem of inadequate blood supplies collected from voluntary blood donors due to the high burden of $\mathrm{HIV}, \mathrm{HBV}, \mathrm{HCV}$, malaria and other infections in the general population (4). Also, access to safe blood is 
limited due to limited resources, inadequate screening facilities and shortage of trained personnel $(5,6)$. As a result, there's a substantial decrease in the number of potential voluntary blood donors and a subsequent shortage of safe blood supplies.

This shortage of blood greatly contributes to high maternal and infant deaths in the country due to pregnancy-related hemorrhage and anemia secondary to malaria. (7-11). We retrospectively analyzed data collected from blood donors from 2005-2015 to assess the burden and determinants of TTIs to help target interventions for safety and increased access to safe blood (8).

\section{Methods}

\section{Study design}

The study was secondary data analysis of a cohort of blood donors from the Malawi Blood Transfusion Service (MBTS) who donated blood over a 10-year period from 2005 to 2015. Blood is collected from all the three regions (Northern, Southern, and Central) of the country from eligible voluntary unpaid blood donors ages $16-45$. Screening for HIV, Hepatitis B and C, syphilis and malaria are performed on all donated blood. HIV is tested using the enzyme-linked immunosorbent assay- ELISA (p24 antigen and antibodies for HIV 1 and 2), hepatitis B virus (HbsAg), HCV (anti-HCV antibodies) and syphilis (Treponema pallidum hemagglutination-TPHA test). Blood films are screened for malaria parasites. All routine tests are integrated and performed using standard operating procedures (SOPs) to minimize errors $(12,13)$ in Blantyre. Additional tests performed on donated blood included ABO blood grouping and rhesus $\mathrm{D}(\mathrm{RhD})$ antigens.

\section{Measures of outcomes and exposures}

The four outcomes were TTI, HIV, HCV, and HBV. TTI was defined as a donor who tested positive for either HIV, HBV or HCV after the first donation. The exposure variables were socio-demographic characteristics, which were age, sex, marital status, employment status, region of residence, and clinical information of donors (blood group, Other TTIs). Marital status was categorised into married, not married (to include the divorced, separated and widowed). The different forms of occupation, renamed employment status, were grouped into three categories namely employed (included students and other formal employment), unemployed and 'unknown' to include those whose form of employment did not identify with any form of employment in the Labour market. 'Other TTIs' was generated as a categorical variable to include malaria, syphilis and 'none' if donor was negative for either malaria or syphilis. The entries for districts in the database was grouped into the Central, Southern and Northern regions of Malawi, renamed as region of residence. A fourth category 'unknown' was generated to include those districts that did not identify with any of the three regions of Malawi. 


\section{Statistical analysis}

The analysis consisted of a total of 47,075 blood donors aged $16-65$, who contributed at least two blood donations into the study, and were negative for HIV, HCV or HBV at the time of testing with complete records on their HIV, HCV or HBV status. The data were cleaned, checked for consistency and duplicate records. A unique ID (donor ID) for each donor was generated based on the number of blood donations contributed throughout the study period. All donors with donor ID less that one (that is only donated once and became infected), below the age of 16 and older than 65, were dropped. Also, donors with missing HIV, HCV or HBV status or who tested positive for HIV, HCV or HBV at first donation were dropped.

Age was computed using the date of TTI testing and date of birth. To account for missing age, firstly, the maximum number of blood donations for each donor was computed and used to compute the average age. Next, the average age was replaced if age was missing and donor ID=1 and where the date of birth was missing, it was imputed with the age multiplied by 365.25 and subtracted from the TTI test date. We described age using median and interquartile range (IQR) and categorized into age groups. All categorical variables were described using percentages and comparisons of factors between those infected and uninfected was done using Pearson Chi-square test and/or Fischer's exact test where there was sparse data.

Disease incidence for the period from 2005-2015 was calculated by dividing the number of new cases over the study period by the total person-years at risk as previously described (14).The cases, personyears at risk and incidence rates per 10,000 person-years were reported with $95 \%$ confidence intervals. The Kaplan-Meier estimates were used to compute the survival time and the overall survival probability as well as the survival probability by sex was calculated as the number of surviving donors divided by the donors at risk. The log-rank test for equality of survivor functions across strata was done. In an initial attempt to identify factors associated with TTI, HIV, HCV or HBV using Cox proportional hazard regression model, three of six predictors violated the proportional hazards assumptions. A modification of the model to include the time-dependent variable for the non-proportional predictors and stratifying on the nonproportional predictors was not practical. As such logistic regression models were used to identify factors independently associated with TTI, HIV, HCV and HBV following consultation with a biostatistician. Analyses were performed with Stata 15.1 software (Stata-Corp. USA).

\section{Results}

\section{Description of Blood Donors}


From 2005 through 2015, 227,442 donor records captured within the MBTS database were retrieved for this study. Of these, only 47,075 donors whose records met all the inclusion criteria were analyzed (Figure 1).

The overall median age was 22 years (IQR=18-22) with majority of donors $(n=21,779 ; 46.3 \%)$ between $20-24$ years. Overall, $84 \%(n=39,568)$ of the donors were males, $91.6 \%(n=43,138)$ not married, $97.6 \%$ $(45,943)$ employed, and $53.1 \%(n=25,014)$ residing in the Southern region. A total of $23,941(50.9 \%)$ donors had type 0 blood group, 42,390 (90\%) reported engaging in high-risk sexual behavior. Malaria infection was common in $20,126(42.8 \%)$ of the donors while a minority $322(0.7 \%)$ was positive for syphilis infection (Table 1).

Table 1: Characteristics of Malawian blood donors who donated blood from 2005-2015 ( $N=47075)$ 


\begin{tabular}{lll}
\hline \multicolumn{1}{c}{ Characteristic } & Number (N) & Percentage (\%) \\
\cline { 2 - 3 } Age (years) & $22 *$ & $(18-22)^{*}$ \\
Age group (\%) & & \\
16-19 & 17464 & 37.1 \\
20-24 & 21779 & 46.3 \\
25-29 & 4343 & 9.2 \\
30-34 & 1663 & 3.5 \\
35-39 & 833 & 1.8 \\
40-44 & 485 & 1.0 \\
45+ & 508 & 1.1 \\
TOTAL & 47075 & 100 \\
Sex (\%) & & \\
Female & 7507 & 16.0 \\
Male & 39568 & 84.0 \\
TOTAL & 47075 & 100 \\
Marital status (\%) & & \\
Married & 3937 & 8.4 \\
Not Married & 43138 & 91.6 \\
TOTAL & 47075 & 100 \\
Employment status (\%) & & \\
Employed & 45943 & 97.6 \\
Unemployed & 714 & 1.5 \\
Unknown & 418 & 0.9 \\
TOTAL & 47075 & 100 \\
Region of residence (\%) & & \\
Central Region & 15344 & 32.6 \\
Northern Region & 6674 & 14.2 \\
Southern Region & 25014 & 53.1 \\
Unknown & 43 & 0.1 \\
TOTAL & 47075 & 100 \\
Blood Group (\%) & & \\
A & 10524 & 22.4 \\
B & 9857 & 20.9 \\
AB & 1925 & 4.1 \\
O & 23126 & 42.8 \\
Missing & 47075 & 100 \\
TOTAL & & \\
Risky sexual behavior (\%) & 47075 & 1.8 \\
Low & & 100 \\
High & & \\
TOTAL & & 10.0 \\
Other TTIs (\%) & & 90.0 \\
None & Syphilis & 100 \\
Malaria & & \\
TOTAL & & 56.6 \\
\hline
\end{tabular}

*Median (Interquartile Range)

TTI, HIV, HCV, and HBV status 
Of the 47,075 registered donors, $3,439(7.3 \%)$ had TTI and the median age was 22 years (IQR=19-23). The frequency of TTI was highest with statistical significance in donors aged 20-24 (49.6\%), males $(90 \%)$, residents of the Southern region (57.4\%), not married (90.7\%), high-risk sexual behavior (88.6\%). Overall, the rate of TTI incidence among the blood donors was 43.4 per 10,000 with the probability of survival being $63 \%$ (Figure 2). By sex, females had a higher survival probability compared to males $(p<0.0001$; Figure 3$)$.

TTI incidence rate was significantly higher among males (46.36 cases per 10,$000 ; 95 \% \mathrm{Cl}=44.76-48.03$ ), $16-19$ age group ( 40.35 cases per 10,$000 ; 95 \% \mathrm{Cl}=38.03-42.81)$, not married $(43.91$ per 10,$000 ; 95 \% \mathrm{Cl}=$ 34.92-43.49), unemployed ( 65.23 per 10,$000 ; 95 \% \mathrm{Cl}=51.43-82.74)$, residing in the Southern region (49.09 per 10,000; 95\% Cl= 46.97-51.30: Figure 4), and low-risk sexual behavior $(49.13$ per 10,000$)$ as shown (Table 2).

Table 2: Donor characteristics and TTI incidence rates by potential risk factors and confounders ( $\mathrm{N}=47$ 075) 


\begin{tabular}{|c|c|c|c|c|c|}
\hline \multirow[t]{2}{*}{ Characteristic } & $\begin{array}{c}\text { TTI Cases }(\mathrm{N}=3 \\
439)\end{array}$ & $\begin{array}{c}p \\
\text { value* }\end{array}$ & $\begin{array}{l}\text { Person } \\
\text { Years }\end{array}$ & $\begin{array}{c}\text { IR (95\% CI) per } 10000 \\
\text { pyear }\end{array}$ & $\begin{array}{c}p \\
\text { value* }\end{array}$ \\
\hline & n (\%) & & & & \\
\hline Age (years) & $22(19-23) *$ & 0.000 & & & \\
\hline Overall & & & 79.24 & $43.40(41.98-44.88)$ & \\
\hline Age group & & 0.000 & & & \\
\hline $16-19$ & $1101(32.0)$ & & 27.28 & 40.35 (38.03-42.81) & 0.000 \\
\hline $20-24$ & $1705(49.6)$ & & 36.02 & 47.33 (45.14-49.63) & \\
\hline $25-29$ & $352(10.2)$ & & 7.07 & $49.81(44.87-55.29)$ & \\
\hline $30-34$ & $168(4.9)$ & & 3.68 & $45.64(39.23-53.09)$ & \\
\hline $35-39$ & $64(1.9)$ & & 2.07 & $30.88(24.17-39.45)$ & \\
\hline $40-44$ & $29(0.8)$ & & 1.47 & $19.70(13.69-28.35)$ & \\
\hline $45+$ & $20(0.6)$ & & 1.64 & $12.22(7.88-18.94)$ & \\
\hline TOTAL & 3439 (100) & & & & \\
\hline Sex & & 0.000 & & & \\
\hline Female & $345(10.0)$ & & 12.50 & $27.59(24.83-30.66)$ & \\
\hline Male & $3094(90.0)$ & & 66.73 & $46.36(44.76-48.03)$ & 0.000 \\
\hline TOTAL & 3439 (100) & & & & \\
\hline Marital status & & 0.045 & & & \\
\hline Married & $319(9.3)$ & & 8.19 & 38.97 (34.92-43.49) & \\
\hline Not Married & 3120 (90.7) & & 71.05 & $43.91(42.40-45.48)$ & 0.000 \\
\hline TOTAL & 3439 (100) & & & & \\
\hline Employment status & & 0.033 & & & \\
\hline Employed & $3334(97.0)$ & & 77.46 & $43.04(41.61-44.53)$ & \\
\hline Unemployed & $68(2.0)$ & & 1.04 & $65.23(51.43-82.74)$ & 0.048 \\
\hline Unknown & $37(1.0)$ & & 0.73 & $50.39(36.51-69.55)$ & \\
\hline TOTAL & 3439 (100) & & & & \\
\hline Region of residence & & 0.000 & & & \\
\hline Central Region & $1153(33.5)$ & & 26.12 & 44.14 (41.67-46.77) & \\
\hline Northern Region & $308(9.0)$ & & 12.80 & $24.06(21.52-26.90)$ & \\
\hline Southern Region & $1975(57.4)$ & & 40.24 & 49.09 (46.97-51.30) & 0.000 \\
\hline Unknown & $3(0.1)$ & & 0.08 & $37.55(12.11-116.42)$ & \\
\hline TOTAL & 3439 (100) & & & & \\
\hline Blood group & & 0.301 & & & \\
\hline A & $820(23.8)$ & & 17.73 & $46.25(43.19-49.52)$ & 0.158 \\
\hline B & $702(20.4)$ & & 16.40 & $42.81(39.76-46.10)$ & \\
\hline $\mathrm{AB}$ & $135(4.0)$ & & 3.25 & $41.56(35.11-49.20)$ & \\
\hline $\mathrm{O}$ & $1723(50.1)$ & & 40.29 & $42.76(40.80-44.83)$ & \\
\hline Missing & $59(1.7)$ & & 1.57 & 37.68 (29.20-48.63) & \\
\hline TOTAL & 3439 (100) & & & & \\
\hline $\begin{array}{l}\text { Risky sexual } \\
\text { behavior }\end{array}$ & & 0.004 & & & \\
\hline Low & $391(11.4)$ & & 7.96 & $49.13(44.50-54.25)$ & 0.004 \\
\hline High & $3048(88.6)$ & & 71.28 & $42.76(41.27-44.31)$ & \\
\hline TOTAL & 3439 (100) & & & & \\
\hline Other TTIs & & 0.716 & & & \\
\hline None & $1934(56.2)$ & & 49.29 & $39.24(37.53-41.03)$ & \\
\hline Syphilis & $27(0.8)$ & & 0.63 & $42.54(29.17-62.03)$ & \\
\hline Malaria & $1478(43.0)$ & & 29.32 & $50.42(47.91-53.05)$ & 0.000 \\
\hline TOTAL & 3439 (100) & & & & \\
\hline
\end{tabular}

*Median (Interquartile Range); $p$-value= obtained by Pearson Chi-square for statistical differences within groups, significant at $p<0.05$. All significant values are in bold face; $\mathrm{IR}=$ Incidence rate; $\mathrm{Cl}=$ Confidence intervals 
For HIV, 818 (1.7\%) were HIV infected with a median age of 22 years (IQR=19-23). The frequency of HIV was highest with statistical significance in donors aged $20-24(47.2 \%)$, male (87.4\%) residents of the Southern region (54.9\%), not married (88\%), and high-risk sexual behavior (87.3\%). Overall, the rate of HIV incidence among donors was 10.32 per $10,000(95 \% \mathrm{Cl}=9.64-11.06)$ with the probability of survival of $84 \%$ (Figure 2). By sex, there was no significant difference in the survival probability ( $p>0.05$; Figure 3 ). HIV incidence rate was significantly higher in $16-19$ age group $(91.26$ cases per 10,$000 ; 95 \% \mathrm{Cl}=80.60-$ 103.33), not married (101.33 per 10,000; $95 \% \mathrm{Cl}=94.20-109.01)$, residing in the Southern (111.59 per $10,000 ; 95 \% \mathrm{Cl}=101.73-122.41)$ and Central (115.24 per 10,000; $95 \% \mathrm{Cl}=102.93-129.02)$ regions, lowrisk sexual behavior ( 130.68 per 10,$000 ; 95 \% \mathrm{Cl}=107.83-158.38)$, and syphilis co-infection (252.08 per 10,000; $95 \% \mathrm{Cl}=154.44-411.48)$ as shown (Table 3 ).

Table 3: Donor characteristics and HIV incidence rates by potential risk factors and confounders $(\mathrm{N}=47$ 075) 


\begin{tabular}{|c|c|c|c|c|c|}
\hline Characteristic & $\begin{array}{l}\text { HIV Cases } \\
(\mathrm{N}=818)\end{array}$ & $\begin{array}{c}p \\
\text { value* }\end{array}$ & $\begin{array}{l}\text { Person } \\
\text { Years }\end{array}$ & $\begin{array}{c}\text { IR (95\% CI) per } 10000 \\
\text { pyear }\end{array}$ & $p$ value* \\
\hline & $\mathrm{n}(\%)$ & & & & \\
\hline Age (years) & $22(19-23) *$ & 0.000 & & & \\
\hline Overall & & & 79.24 & $10.32(09.64-011.06)$ & \\
\hline Age group & & 0.000 & & & \\
\hline $16-19$ & $249(30.4)$ & & 2.73 & 91.26 (80.60-103.33) & 0.000 \\
\hline $20-24$ & $386(47.2)$ & & 3.60 & 107.15 (96.98-118.39) & \\
\hline $25-29$ & $91(11.1)$ & & 0.71 & $128.77(104.85-158.14)$ & \\
\hline $30-34$ & $43(5.3)$ & & 0.37 & $116.81(86.63-157.50)$ & \\
\hline $35-39$ & $18(2.2)$ & & 0.21 & $86.85(54.72-137.86)$ & \\
\hline $40-44$ & $18(2.2)$ & & 0.15 & $122.29(77.05-194.09)$ & \\
\hline $45+$ & $13(1.6)$ & & 0.16 & 79.42 (46.12-136.78) & \\
\hline TOTAL & $818(100)$ & & & & \\
\hline Sex & & 0.008 & & & \\
\hline Female & $103(12.6)$ & & 1.25 & 82.37 (67.91-99.92) & \\
\hline Male & $715(87.4)$ & & 6.67 & 107.14 (99.57-115.29) & 0.552 \\
\hline TOTAL & $818(100)$ & & & & \\
\hline Marital status & & 0.000 & & & \\
\hline Married & $98(12.0)$ & & 0.82 & $119.73(98.22-145.94)$ & \\
\hline Not Married & $720(88.0)$ & & 7.11 & $101.33(94.20-109.01)$ & 0.000 \\
\hline TOTAL & $818(100)$ & & & & \\
\hline Employment status & & 0.607 & & & \\
\hline Employed & $794(97.1)$ & & 7.75 & $102.50(95.62-109.89)$ & \\
\hline Unemployed & $15(1.8)$ & & 0.10 & $143.90(86.75-238.69)$ & 0.662 \\
\hline Unknown & $9(1.1)$ & & 0.07 & $122.58(63.78-235.59)$ & \\
\hline TOTAL & $818(100)$ & & & & \\
\hline Region of residence & & 0.000 & & & \\
\hline Central Region & $301(36.8)$ & & 2.61 & $115.24(102.93-129.02)$ & 0.000 \\
\hline Northern Region & $68(8.3)$ & & 1.28 & $53.12(41.88-67.37)$ & \\
\hline Southern Region & $449(54.9)$ & & 4.02 & 111.59 (101.73-122.41) & 0.000 \\
\hline Unknown & $0(0.0)$ & & 0.01 & 0.00 & \\
\hline TOTAL & $818(100)$ & & & & \\
\hline Blood group & & 0.247 & & & \\
\hline A & $204(24.9)$ & & 1.77 & 115.06 (100.30-131.98) & 0.184 \\
\hline B & 177(21.6) & & 1.64 & 107.94 (93.15-125.07) & \\
\hline $\mathrm{AB}$ & $36(4.4)$ & & 0.32 & 110.83 (79.94-153.65) & \\
\hline $\mathrm{O}$ & $385(47.1)$ & & 4.03 & 95.55 (86.46-105.58) & \\
\hline Missing & $16(2.0)$ & & 0.16 & $102.18(62.60-166.80)$ & \\
\hline TOTAL & $818(100)$ & & & & \\
\hline $\begin{array}{l}\text { Risky sexual } \\
\text { behavior }\end{array}$ & & 0.008 & & & \\
\hline Low & $104(12.7)$ & & 0.80 & $130.68(107.83-158.38)$ & 0.006 \\
\hline High & $714(87.3)$ & & 7.13 & $100.17(93.07-107.79)$ & \\
\hline TOTAL & $818(100)$ & & & & \\
\hline Other TTIs & & 0.000 & & & \\
\hline None & $500(61.1)$ & & 4.93 & 101.45 (92.94-110.74) & \\
\hline Syphilis & $16(2.0)$ & & 0.06 & $252.08(154.44-411.48)$ & 0.003 \\
\hline Malaria I & $302(36.9)$ & & 2.93 & $103.02(92.03-115.31)$ & \\
\hline TOTAL & $818(100)$ & & & & \\
\hline
\end{tabular}

*Median (Interquartile Range); $p$-value= obtained by Pearson Chi-square for statistical differences within groups, significant at $p<0.05$. All significant values are in bold face; $\mathrm{IR}=$ Incidence rate; $\mathrm{Cl}=$ Confidence intervals 
For HCV, 602 (1.3\%) were infected and had a median age of 20 years (IQR=18-22). The frequency was highest with statistical significance in donors aged 20-24 (49.7\%), males (90.5\%), not married (94.5\%), and blood type $\mathrm{O}(43.7 \%)$ as shown (Table 4$)$. Overall, the rate of HCV incidence among donors was 7.60 per $10,000(95 \% \mathrm{Cl}=7.01-8.23)$ with a $93 \%$ probability of survival (Figure 2$)$. By sex, females had a higher survival probability compared to males ( $p=0.0089$; Figure 3 ). HCV incidence rate was significantly higher among males ( 8.17 cases per 10,$000 ; 95 \% \mathrm{Cl}=7.51-8.88), 16-19$ age group (9.02 cases per 10,000; $95 \% \mathrm{Cl}=7.96-1.0 \mathrm{e}+01)$, not married (8.00 per 10,000; $95 \% \mathrm{Cl}=7.38-8.69)$, blood type $\mathrm{AB}$ (8.93 per 10,$000 ; 95 \% \mathrm{Cl}=6.20-12.85)$, and syphilis co-infection (14.18 per 10,$000 ; 95 \% \mathrm{Cl}=7.38-27.25)$ as shown (Table 4).

Table 4: Donor characteristics and HCV incidence rates by potential risk factors and confounders $(\mathrm{N}=47$ 075) 


\begin{tabular}{|c|c|c|c|c|c|}
\hline \multirow[t]{2}{*}{ Characteristic } & $\begin{array}{l}\text { HCV Cases } \\
(\mathrm{N}=602)\end{array}$ & $\begin{array}{c}p \\
\text { value* }\end{array}$ & $\begin{array}{l}\text { Person } \\
\text { Years }\end{array}$ & $\begin{array}{c}\text { IR (95\% CI) per } 10000 \\
\text { pyear }\end{array}$ & $\begin{array}{c}p \\
\text { value* }\end{array}$ \\
\hline & $n(\%)$ & & & & \\
\hline Age (years) & $20(18-22) *$ & 0.070 & & & \\
\hline Overall & & & 79.24 & $7.60(7.01-8.23)$ & \\
\hline Age group & & 0.000 & & & \\
\hline $16-19$ & $246(41.0)$ & & 27.28 & $9.02(7.96-1.0 \mathrm{e}+01)$ & 0.000 \\
\hline $20-24$ & $299(49.7)$ & & 36.02 & $8.30(7.41-9.30)$ & \\
\hline $25-29$ & $37(6.1)$ & & 7.07 & $5.24(3.79-7.23)$ & \\
\hline $30-34$ & $13(2.1)$ & & 3.68 & $3.53(2.05-6.08)$ & \\
\hline $35-39$ & $3(0.5)$ & & 2.07 & $1.45(0.47-4.49)$ & \\
\hline $40-44$ & $3(0.5)$ & & 1.47 & $2.04(0.66-6.32)$ & \\
\hline $45+$ & $1(0.1)$ & & 1.64 & $0.61(0.09-4.34)$ & \\
\hline TOTAL & $602(100)$ & & & & \\
\hline Sex & & 0.000 & & & \\
\hline Female & $57(9.5)$ & & 12.50 & $4.56(3.52-5.91)$ & \\
\hline Male & $545(90.5)$ & & 66.73 & $8.17(7.51-8.88)$ & 0.009 \\
\hline TOTAL & $602(100)$ & & & & \\
\hline Marital status & & 0.010 & & & \\
\hline Married & $33(5.5)$ & & 8.19 & $4.03(2.87-5.67)$ & \\
\hline Not Married & $569(94.5)$ & & 71.05 & $8.00(7.38-8.69)$ & 0.000 \\
\hline TOTAL & $602(100)$ & & & & \\
\hline Employment status & & 0.686 & & & \\
\hline Employed & $591(98.2)$ & & 77.46 & $7.63(7.04-8.27)$ & 0.265 \\
\hline Unemployed & $8(1.3)$ & & 1.04 & 7.67 (3.84-15.35) & \\
\hline Unknown & $3(0.5)$ & & 0.73 & 4.09 (1.32-12.67) & \\
\hline TOTAL & $602(100)$ & & & & \\
\hline Region of residence & & 0.084 & & & \\
\hline Central Region & $220(36.5)$ & & 26.12 & $8.42(7.38-9.61)$ & 0.294 \\
\hline Northern Region & $72(12.0)$ & & 12.80 & $5.62(4.46-7.09)$ & \\
\hline Southern Region & $309(51.3)$ & & 40.24 & 7.68 (6.87-8.59) & 0.294 \\
\hline Unknown & $1(0.2)$ & & 0.08 & $12.52(1.76-88.85)$ & \\
\hline TOTAL & $602(100)$ & & & & \\
\hline Blood group & & 0.001 & & & \\
\hline A & $156(25.9)$ & & 17.73 & $8.80(7.52-10.29)$ & 0.002 \\
\hline B & $135(22.4)$ & & 16.40 & $8.23(6.95-9.75)$ & 0.002 \\
\hline $\mathrm{AB}$ & $29(4.8)$ & & 3.25 & $8.93(6.20-12.85)$ & 0.002 \\
\hline $\mathrm{O}$ & $263(43.7)$ & & 40.29 & $6.53(5.78-7.37)$ & \\
\hline Missing & $19(3.2)$ & & 1.57 & $12.13(7.74-19.02)$ & 0.002 \\
\hline TOTAL & $602(100)$ & & & & \\
\hline $\begin{array}{l}\text { Risky sexual } \\
\text { behavior }\end{array}$ & & 0.054 & & & \\
\hline Low & $74(12.3)$ & & 7.96 & $9.30(7.40-11.68)$ & 0.061 \\
\hline High & $528(87.7)$ & & 71.28 & $7.41(6.80-8.07)$ & \\
\hline TOTAL & $602(100)$ & & & & \\
\hline Other TTIs & & 0.034 & & & \\
\hline None & $349(58.0)$ & & 49.29 & $7.08(6.38-7.86)$ & \\
\hline Syphilis & $9(1.5)$ & & 0.63 & $14.18(7.38-27.25)$ & 0.037 \\
\hline Malaria & $244(40.5)$ & & 29.32 & $8.32(7.34-9.44)$ & \\
\hline TOTAL & $602(100)$ & & & & \\
\hline
\end{tabular}

*Median (Interquartile Range); $p$-value= obtained by Pearson chi2 or Fisher's exact test to compare frequencies between groups, significant at $p<0.05$. All significant values are in bold face; IR= Incidence rate; $\mathrm{Cl}=$ Confidence intervals 
Similarly, 1,238 (2.6\%) were infected with HBV and the median age was 22 years (IQR=19-22). The frequency of HBV was highest with statistical significance in donors aged 20-24 (50.6\%), males (89.2\%), and residents of the Southern region (50.2\%) as shown (Table 5). Overall, the rate of HBV incidence among donors was 15.62 per $10,000(95 \% \mathrm{Cl}=14.78-16.52)$ with $83 \%$ probability of survival (Figure 2$)$. By sex, females had a higher survival probability compared to males $(p=0.0089$; Figure 3$)$. HBV incidence rate was significantly higher among males (16.54 cases per 10,$000 ; 95 \% \mathrm{Cl}=15.60-17.55), 16-19$ age group ( 15.76 cases per 10,$000 ; 95 \% \mathrm{Cl}=14.34-17.32)$, not married $(16.20$ per 10,$000 ; 95 \% \mathrm{Cl}=15.29-$ 17.16), residing in the Central region (18.53 per 10,000; $95 \% \mathrm{Cl}=16.95-20.25)$, and syphilis co-infection (23.63 per 10,$000 ; 95 \% \mathrm{Cl}=15.95-18.97)$ as shown (Table 5).

Table 5: Donor characteristics and HBV incidence rates by potential risk factors and confounders $(\mathrm{N}=47$ 075) 


\begin{tabular}{|c|c|c|c|c|c|}
\hline Characteristic & $\begin{array}{c}\text { HBV Cases }(\mathrm{N}=1 \\
\text { 238) }\end{array}$ & $\begin{array}{c}p \\
\text { value* }\end{array}$ & $\begin{array}{l}\text { Person } \\
\text { Years }\end{array}$ & $\begin{array}{c}\text { IR (95\% CI) per } 10000 \\
\text { pyear }\end{array}$ & $\stackrel{p}{p}$ \\
\hline & n (\%) & & & & \\
\hline Age (years) & $22(19-22) *$ & 0.215 & & & \\
\hline Overall & & & 79.24 & $15.62(14.78-16.52)$ & \\
\hline Age group & & 0.005 & & & \\
\hline $16-19$ & $430(34.7)$ & & 27.28 & $15.76(14.34-17.32)$ & 0.000 \\
\hline $20-24$ & $626(50.6)$ & & 36.02 & 17.38 (16.07-18.79) & \\
\hline $25-29$ & $101(8.2)$ & & 7.07 & 14.29 (11.76-17.37) & \\
\hline $30-34$ & $52(4.2)$ & & 3.68 & $14.13(10.76-18.54)$ & \\
\hline $35-39$ & $16(1.3)$ & & 2.07 & $07.72(04.73-12.60)$ & \\
\hline $40-44$ & $6(0.5)$ & & 1.47 & 04.08 (01.83-09.07) & \\
\hline $45+$ & $7(0.6)$ & & 1.64 & $04.28(02.04-08.97)$ & \\
\hline TOTAL & 1238 (100) & & & & \\
\hline Sex & & 0.000 & & & \\
\hline Female & $134(10.8)$ & & 12.50 & $10.72(09.05-12.69)$ & \\
\hline Male & $1104(89.2)$ & & 66.73 & $16.54(15.60-17.55)$ & 0.009 \\
\hline TOTAL & $1238(100)$ & & & & \\
\hline Marital status & & 0.085 & & & \\
\hline Married & $87(7.0)$ & & 8.19 & $10.63(08.61-13.11)$ & \\
\hline Not Married & $1151(93.0)$ & & 71.05 & $16.20(15.29-17.16)$ & 0.000 \\
\hline TOTAL & $1238(100)$ & & & & \\
\hline Employment status & & 0.404 & & & \\
\hline Employed & $1207(97.5)$ & & 77.46 & 15.58 (14.73-16.49) & 0.127 \\
\hline Unemployed & $23(1.9)$ & & 1.04 & $22.06(14.66-33.20)$ & 0.127 \\
\hline Unknown & $8(0.6)$ & & 0.73 & $10.90(05.45-21.79)$ & \\
\hline TOTAL & 1238 (100) & & & & \\
\hline Region of residence & & 0.000 & & & \\
\hline Central Region & $484(39.1)$ & & 26.12 & 18.53 (16.95-20.25) & 0.000 \\
\hline Northern Region & 133 (10.7) & & 12.80 & 10.39 (08.77-12.31) & \\
\hline Southern Region & $621(50.2)$ & & 40.24 & $15.43(14.27-16.70)$ & \\
\hline Unknown & $0(0.00)$ & & 0.08 & 00.00 & \\
\hline TOTAL & 1238 (100) & & & & \\
\hline Blood group & & 0.505 & & & \\
\hline A & $290(23.4)$ & & 17.73 & 16.36 (14.57-18.35) & 0.389 \\
\hline B & 246 (19.9) & & 16.40 & 15.00 (13.24-16.99) & \\
\hline $\mathrm{AB}$ & $47(3.8)$ & & 3.25 & $14.47(10.87-19.26)$ & \\
\hline $\mathrm{O}$ & $639(51.6)$ & & 40.29 & $15.86(14.68-17.14)$ & 0.389 \\
\hline Missing & $16(1.3)$ & & 1.57 & $10.22(06.26-16.68)$ & \\
\hline TOTAL & $1238(100)$ & & & & \\
\hline $\begin{array}{l}\text { Risky sexual } \\
\text { behavior }\end{array}$ & & 0.057 & & & \\
\hline Low & $143(11.5)$ & & 7.65 & $17.97(15.25-21.16)$ & 0.060 \\
\hline High & $1095(88.5)$ & & 71.28 & $15.36(14.47-16.30)$ & \\
\hline TOTAL & 1238 (100) & & & & \\
\hline Other TTIs & & 0.047 & & & \\
\hline None & $713(57.6)$ & & 49.29 & 14.47 (13.44-15.57) & \\
\hline Syphilis & $15(1.2)$ & & 0.63 & $23.63(14.25-39.20)$ & 0.004 \\
\hline Malaria & $510(41.2)$ & & 29.32 & 17.40 (15.95-18.97) & 0.004 \\
\hline TOTAL & $1238(100)$ & & & & \\
\hline
\end{tabular}

*Median (Interquartile Range); $p$-value= obtained by Pearson Chi-square or Fischer's exact test to compare frequencies between groups, significant at $p<0.05$. All significant values are in bold face; IR= Incidence rate; $\mathrm{Cl}=$ Confidence intervals 
In the multivariate analysis, the odds of TTI were higher in males ( $\mathrm{OR}=1.65 ; 95 \% \mathrm{Cl}=1.46-1.84)$, age groups $16-19,20-24,25-29,30-34$, and $35-39(\mathrm{aOR}=1.82,95 \% \mathrm{Cl}=1.15-2.89 ; \mathrm{aOR}=2.15,95 \% \mathrm{Cl}=$ $1.36-3.41 ; \mathrm{aOR}=2.13,95 \% \mathrm{Cl}=1.33-3.39 ; \mathrm{aOR}=2.69,95 \% \mathrm{Cl}=1.67-4.34 ;$ and $\mathrm{aOR}=2.00,95 \% \mathrm{Cl}=$ 1.19 - 3.35, respectively). Donors who were married and engaged in high-risk sexual behavior had a 2fold increased risk of TTI (Table 6).

Table 6: Predictors of TTI in blood donors: multivariate model*

\begin{tabular}{|c|c|c|}
\hline \multirow[t]{2}{*}{ Characteristic } & \multicolumn{2}{|l|}{ Multivariate Analysis } \\
\hline & Adjusted Odds Ratio (95\% CI) & $p$ value* \\
\hline \multicolumn{3}{|l|}{ Age group } \\
\hline $16-19$ & $1.82(1.15-2.89)$ & 0.011 \\
\hline $20-24$ & $2.15(1.36-3.41)$ & 0.001 \\
\hline $25-29$ & $2.13(1.33-3.39)$ & 0.002 \\
\hline $30-34$ & $2.69(1.67-4.34)$ & 0.000 \\
\hline $35-39$ & $2.00(1.19-3.35)$ & 0.008 \\
\hline $40-44$ & $1.54(0.86-2.76)$ & 0.148 \\
\hline $45+$ & REF & \\
\hline \multicolumn{3}{|l|}{ Sex } \\
\hline Female & REF & \\
\hline Male & 1.65 (1.47-1.85) & 0.000 \\
\hline \multicolumn{3}{|l|}{ Marital status } \\
\hline Married & $1.92(1.38-2.68)$ & 0.000 \\
\hline Not Married & REF & \\
\hline \multicolumn{3}{|l|}{ Region of residence } \\
\hline Central Region & REF & \\
\hline Northern Region & $0.64(0.57-0.73)$ & 0.000 \\
\hline Southern Region & $1.08(1.00-1.16)$ & 0.049 \\
\hline Unknown & $0.97(0.30-3.16)$ & 0.963 \\
\hline \multicolumn{3}{|l|}{ Blood group } \\
\hline A & REF & \\
\hline B & $0.90(0.81-1.00)$ & 0.062 \\
\hline $\mathrm{AB}$ & $0.89(0.74-1.08)$ & 0.228 \\
\hline $\mathrm{O}$ & $0.91(0.84-1.00)$ & 0.039 \\
\hline Missing & $0.96(0.73-1.27)$ & 0.793 \\
\hline \multicolumn{3}{|c|}{ maritalstatus\#riskyess } \\
\hline married\#high & $0.47(0.34-0.66)$ & 0.000 \\
\hline not married\#high & $0.92(0.82-1.03)$ & 0.163 \\
\hline
\end{tabular}

* Model adjusted for the variables shown; significant at $p<0.05$. All significant values are in bold face 
For HIV, male sex, being married and syphilis co-infection was associated with a 1.25 -fold $(95 \% \mathrm{Cl}=1.02$ - 1.54), 1.49 -fold ( $95 \% \mathrm{Cl}=1.21-1.85)$, and 2.62 -fold $(95 \% \mathrm{Cl}=1.57-4.38)$ increased odds of HIV, respectively (Table 7). Regarding HCV, male sex, syphilis co-infection and age groups 16-19 and 20-24 were associated with a 1.90 -fold $(95 \% \mathrm{Cl}=1.44-2.51), 2.03$-fold $(95 \% \mathrm{Cl}=1.04-3.98), 7.91$-fold ( $95 \%$ $\mathrm{Cl}=1.11-56.54)$, and a 7.22 -fold $(95 \% \mathrm{Cl}=1.01-51.50)$ increased odds of HCV, respectively (Table 7). Similarly, the independent predictors of HBV were age groups 20-24 (associated with a 2.14-fold increased odds of incident $\mathrm{HBV} ; 95 \% \mathrm{Cl}=1.01-4.53$ ) and 30-34 (associated with a 2.26-fold increased odds of incident $\mathrm{HBV} ; 95 \% \mathrm{Cl}=1.02-5.01$ ), male sex (associated with a 1.53-fold increased odds of incident $\mathrm{HBV} ; 95 \% \mathrm{Cl}=1.27-1.84)$ and syphilis co-infection (1.71-fold increased odds of incident $\mathrm{HBV}$; $95 \% \mathrm{Cl}=1.01-2.89)($ Table 7$)$.

Table 7: Predictors of HIV, HCV and HBV in blood donors: multivariate model* 


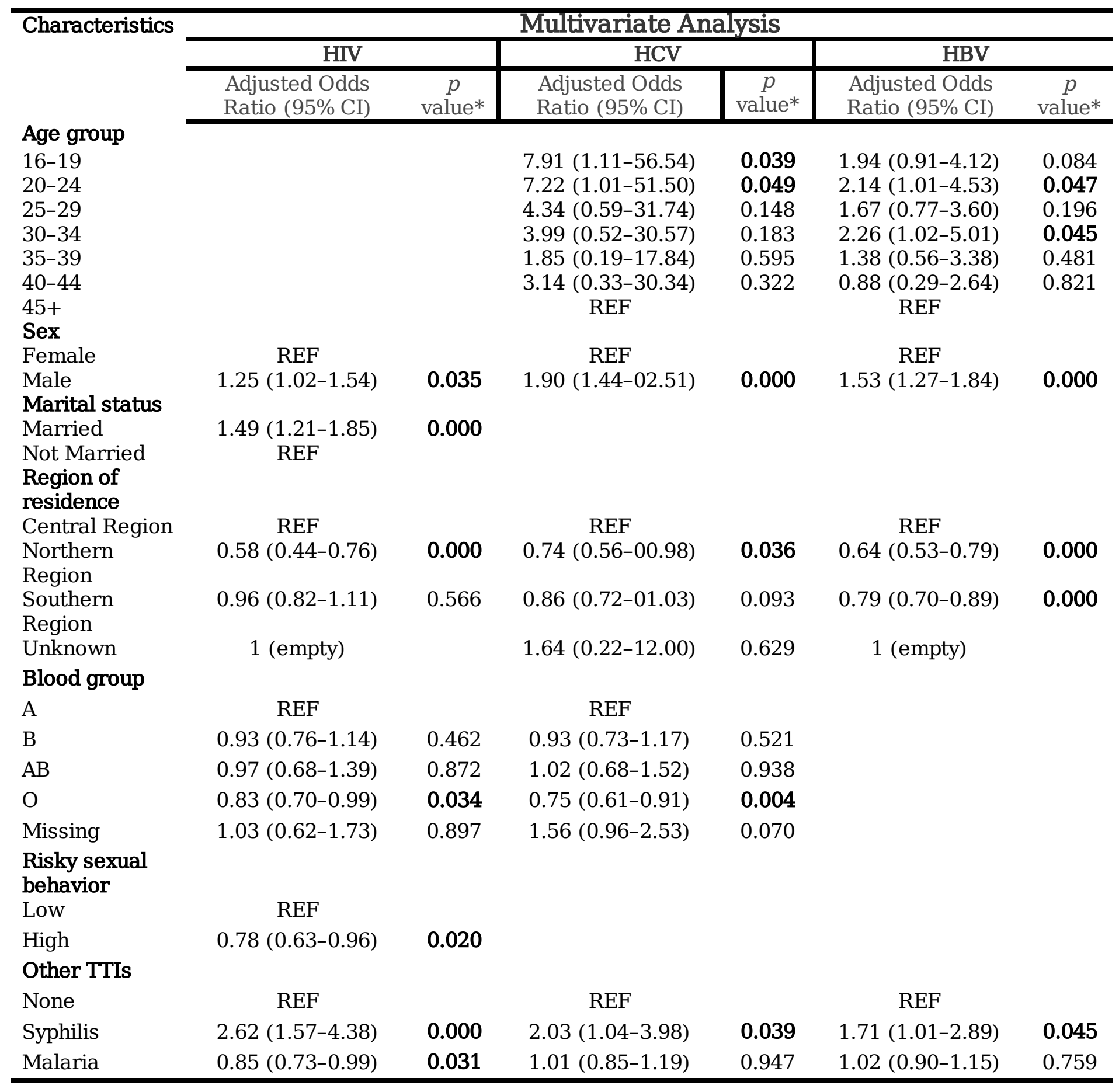

* Model adjusted for the variables shown; significant at $p<0.05$. All significant values are in bold face

\section{Discussion}

\section{Summary of findings}

The overall incidence rate of TTI in the blood donors was 43 per 10,000 with a higher incident rate at 15 per 10,000 in HBV-infected donors followed by HIV with 10 per 10,000 and HCV with 7 per 10,000. The observed TTI incidence was significantly higher among donors aged 16-19 years, males, unmarried donors, unemployed, Southern region, high-risk sexual behavior, and malaria-positive donors. Similarly, 
the observed HIV, HBV and HCV incidence was higher among donors aged 16-19, donors not married, and co-infected with syphilis.

In this study, we established that below 40 years of age groups, male sex, and being married were significantly associated with TTI incidence. In particular, age group 20-24 was commonly associated with incident HCV and HBV. More so, male sex and syphilis co-infection remained a common risk factors for incident HIV, HCV and HBV infections. As observed, married status was a significant risk factor for HIV. The frequency of TTI markers in this study was significantly associated with sex with a higher incidence reported in male donors. Similarly, the frequency of transfusion transmissible HIV, HCV and HBV markers was also found to be significantly associated with sex with a higher incidence reported in male donors.

According to our findings, the overall incidence of HIV among blood donors was much lower compared to other studies conducted elsewhere in Africa $(4,15-18)$. However, it was higher than the $1.37 \%$ obtained in Nigeria (19), 0.014 \% in Libya (20), 0.6\% in Eritrea (21), and 0.00\% Egypt (22). Overall, HCV incidence was $1.28 \%$, which was higher than findings from previous findings within Southern Africa $(23,24)$. Although our finding compares favourably to a study by Biadgo et al. (25) in North West Ethiopia, the incidence is lower when compared to the observed $1.5 \%$ in Tanzania (26), 0.32\% in Ethiopia (27), 4.8\% in Cameroon (28), and 3.4\% in Sudan (29). This result agrees with the common knowledge that HCV poses less risk to blood transfusion in Southern parts of Africa by virtue of its low prevalence (30). The use of serological tests for HCV screening as opposed to nucleic acid testing (NAT) may have accounted for the low incidence in the population due to possible false negative results, which is mostly common in immunocompromised individuals, and the presence of a 45-68 window period. Therefore, this result may have underestimated the frequency of TTIs among donors in the donor population $(21,41)$. We therefore suggest to the MBTS to continue monitoring for the incidence of TTI markers in the donor population through use of more contextualized screening questionnaires and the use of NAT in addition to serological tests.

The most dominant marker for TTI through the study duration was HBV $(2.63 \%)$ similar to a recent finding among blood donors in Malawi (13). The reported incidence is comparable to what was reported in Eritrea (21) but lower than results obtained from other studies $(16,31)$. In SSA, HBV among blood donors in high and this has been attributed to the generally high prevalence (8\%) and endemicity of this pathogen in the region (30). These results support the fact that HBV in the general population is high (32). Remarkably, incident HBV exhibited a statistically significant association with age groups 20-24 and $30-34$, male sex, region of residence and syphilis co-infection. Behavioural, cultural and socioeconomic disparities associated with belonging to these groupings may explain the detected variation. In 
particular, the significant association of incident HBV and HCV in donors aged 20-24 years may be linked to early sexual activity, early marriage, among others in this age group.

The incidence of TTI markers in the donor population in the assessed age groups was different. This result is in line with findings from other studies $(21,37)$. The frequency of TTI markers was substantially higher in the age groups $16-19,20-24,25-29,30-34$, and $35-39$. Donors aged 40 and above had the lowest TTI frequency. The findings indicate that youths constitute a major population of blood donors. Looking at the age structure of the Malawi, $16-24$ and $25-54$ years represent $20.58 \%$ and $27.41 \%$ of the population, respectively (38). These age groups fulfil the selection criteria for blood donation compared to the older age groups. Also, behavioral characteristics unique to these age categories may explain the high incidence of TTI in these age groups. Majority of blood donors in Malawi are young men between the ages 16-25 years. This may in part, explain why the country struggles to meet its annual blood need. Therefore, the MBTS should institute mechanisms to widen the donor age group by massively recruiting more people of older ages.

The male majority of blood donors observed is consistent with previous studies in Africa $(18,28,33)$. In a study by Mohammed and Bekele (34) conducted at Eastern Ethiopia, a similar association was observed (11.6\% males compared to 3.8\% females). In their study, Ataro et al. (18) noted a higher TTI prevalence among male donors (7.46\%) compared to a $4.76 \%$ in female donors. According to them, "this might be due to some risk behaviors such as outside socialization, multiple sex relationships frequently observed in males, and fewer males donating blood, which translates to fewer female screening compared to males" (18). Siraj et al. (21) attributes this unbalanced male-to-female incident TTI ratio to behavioral, religious and socio-cultural drivers of high-risk sexual behavior that are typically found in conventional societies. Another possible explanation as proposed by Tenthani et al. (35) is females are better diagnosed due to antenatal care. As a result, more female blood donors may be conscious of their seronegative status for some of the TTIs. According to Tagny and Owusu-Ofori (36), some physiological status of women such as menstruation, pregnancy and breast-feeding, which occasionally, may prohibit the female gender from donating blood.

Regional disparity in TTI incidence was also observed; donors from the Central and Southern regions had higher incidence compared to the northerners. This may be attributed to cultural norms such as initiation ceremonies and rituals that have been associated with a high rate of unprotected sex, multiple and concurrent sex partners, and being married, all which have been shown to be potential drives for HIV infection $(39,40)$. Also, regional variations in the proportion of people living in informal settlements in these regions is high, which could also contribute to a high-risk sexual behavior and a subsequent increase in incident TTI. Our findings highlight the need for the MBTS to implement more stringent donor 
selection and blood screening procedures to improve the safety of blood supply. This can be achieved through the recruitment of more females, unmarried people, and more donors from the Northern region for blood donation to ensure the safety of donated blood and increase blood volume supply thereby combating the ongoing crisis of blood shortage.

\section{Limitations of the study}

A few limitations are noteworthy; firstly, this study was retrospective in design and therefore the analysis relied solely on the data collection, recording, and the screening systems used by the blood bank. Secondly, because it's retrospective nature, a range of potential co-morbidities that might be associated with TTIs was not evaluated. Despite the limitations, the sample size used for this study was large providing enough statistical power to highlight the incidence and risk factors of major TTIs in the Malawian donor population.

\section{Conclusion}

The overall incidence of TTIs in the Malawian blood donor population is low. Nonetheless, the risk of TTIs remains a problem. The incidence of TTI is more frequent in the younger age groups who constitute the majority of blood donors. An increased trend in the rate of TTI was observed with increasing age up to age 29. HCV incidence on the other hand decreased with increasing age. The most common TTIs is HBV followed by HIV and HCV. The risk of TTI is less likely for donors residing in the Northern region who constituted a minority of the donor population. The risk factors for TTIs are age, particularly in the younger age groups; male sex and married status. Syphilis is a common risk factor for HIV, HCV and HBV. Future research can be done to estimate the volume of blood lost due to the incidence of TTIs in the donor population.

\section{List Of Abbreviations}

HBVHepatitis B Virus

HCVHepatitis C Virus

HCCHepatocellular Carcinoma

HIVHuman Immune Deficiency Virus

IDUnjection Drug Use

IQRInterquartile Range

IRIncident Rate 
MBTSMalawi Blood Transfusion Service

MERMonitoring, Evaluation and Reporting

MWHSWMMen Who Have Sex with Men

NATNucleic Acid Testing

NHSRCNational Health Sciences Research Committee

PWIDPeople Who Inject Drugs

STDSexually Transmitted Disease

SOPStandard Operating Procedure

TT/Transfusion Transmissible Infection

WHOWorld Health Organization

\section{Declarations}

\section{Ethics approval}

The study was approved by the Human Research Ethics Committee (Medical), University of the Witwatersrand, South Africa (Clearance Certificate Number: M181008) and the Malawi National Health Sciences Research Committee- NHSRC (Clearance Certificate Number: 2198).

\section{Availability of data and materials}

The data that support the findings of this study are available from the Malawi Blood Transfusion Services, but restrictions apply to the availability of these data, which were used under license for the current study, and so are not publicly available. Data are however available from the authors upon reasonable request and with permission of the MBTS Director.

\section{Competing interest}

The authors declare no conflict of interest.

\section{Funding}

Not applicable 


\section{Author's contributions}

Constance N Wose Kinge conceptualized the research questions, analyzed the data, and wrote the manuscript; Juliana Kagura and Charles Chasela reviewed the manuscript, Bridon M'baya and Steven Njolomole supervised the blood and data collection processes.

\section{Acknowledgements}

We acknowledge the MBTS for their permission to use the Malawi blood donors' data

\section{References}

1.Platt L, Easterbrook P, Gower E, McDonald B, Sabin K, McGowan C, et al. Prevalence and burden of HCV co-infection in people living with HIV: a global systematic review and meta-analysis. The Lancet Infectious diseases. 2016;16(7):797-808.

2.Soriano V, Young B, Reau N. Report from the International Conference on Viral Hepatitis - 2017. AIDS reviews. 2018;20(1):58-70.

3.Gallaher JR, Mulima G, Kopp D, Shores CG, Charles AG. Consequences of centralised blood bank policies in sub-Saharan Africa. The Lancet Global health. 2017;5(2):e131-e2.

4.Xie DD, Li J, Chen JT, Eyi UM, Matesa RA, Obono MM, et al. Seroprevalence of Human Immunodeficiency Virus, Hepatitis B Virus, Hepatitis C Virus, and Treponema pallidum Infections among Blood Donors on Bioko Island, Equatorial Guinea. PLoS One. 2015;10(10):e0139947.

5.Osaro E, Charles AT. The challenges of meeting the blood transfusion requirements in Sub-Saharan Africa: the need for the development of alternatives to allogenic blood. Journal of blood medicine. 2011;2:7-21.

6.Loua A, Sonoo J, Musango L, Nikiema JB, Lapnet-Moustapha T. Blood Safety Status in WHO African Region Countries: Lessons Learnt from Mauritius. Journal of blood transfusion. 2017;2017:1970479.

7.Colbourn T, Lewycka S, Nambiar B, Anwar I, Phoya A, Mhango C. Maternal mortality in Malawi, 19772012. BMJ open. 2013;3(12):e004150.

8.Demir M, Phiri S, Kaiser R, Chaweza T, Neuhann F, Tweya H, et al. HIV/Hepatitis C Virus Co-infection among Adults Beginning Antiretroviral Therapy, Malawi. Emerging infectious diseases. 2016;22(11):2018-20.

9. Hussein N, Haj S, Almizori L, Taha A. The prevalence of hbv and hcv among blood donors attending blood bank in Duhok city, Kurdistan region, Iraq. Int J Infect. 2016. 
10.Mtengezo J, Lee H, Ngoma J, Kim S, Aronowitz T, DeMarco R, et al. Knowledge and Attitudes toward HIV, Hepatitis B Virus, and Hepatitis C Virus Infection among Health-care Workers in Malawi. Asia-Pacific journal of oncology nursing. 2016;3(4):344-51.

11.Ronald M. Report on the Confidential Enquiry into Maternal Deaths in Malawi (2008-2012). In: Health Mo, editor. Malawi2016.

12.Kongnyuy EJ, van den Broek N. Availability and safety of blood for transfusion in three districts in Malawi. Tropical medicine and health. 2008:0811070036-.

13.Bridon M'baya VJ, Vincent Samuel, Robert M'bwana, and Charles Mangani. Seroprevalence and trends in transfusion transmissible infections among voluntary non-remunerated blood donors at the Malawi Blood Transfusion Service-a time trend study. Malawi Medical Journal. 2019;31(2):118-25.

14.Passannante Maria SA, Ahamed Nisha, editor. Basic Epidemiology

for Tuberculosis Program Staff 2nd Edition. 2nd ed: New Jersey Medical School Global Tuberculodsis Institute; 2005.

15.Omran D, Hussein EA, Nagib M. Safety of blood transfusion: an Egyptian study. Journal of Infectious Diseases and Therapy. 2013.

16.Arshad A, Borhany M, Anwar N, Naseer I, Ansari R, Boota S, et al. Prevalence of transfusion transmissible infections in blood donors of Pakistan. BMC Hematology. 2016;16(1):27.

17.Bisetegen FS, Bekele FB, Ageru TA, Wada FW. Transfusion-transmissible infections among voluntary blood donors at Wolaita Sodo University teaching referral hospital, South Ethiopia. Canadian Journal of Infectious Diseases and Medical Microbiology. 2016;2016.

18.Ataro Z, Urgessa F, Wasihun T. Prevalence and Trends of Major Transfusion Transmissible Infections among Blood Donors in Dire Dawa Blood bank, Eastern Ethiopia: Retrospective Study. Ethiopian journal of health sciences. 2018;28(6):701-10.

19.Akinleye O, Olaniyan J, Akintola J, Okoye C, Eke C. Blood safety and prevalence of transfusion transmissible viral infections among blood donors in Lagos, Nigeria. Int J Trop Med. 2013;8(5-6):113-8.

20.Ali MS, Qowaider S, Moftah S. Seroprevalence rates of transfusion-transmitted infections among blood donors in northeast of Libya. J Sci Humanit. 2014;19:1-7.

21.Siraj N, Achila OO, Issac J, Menghisteab E, Hailemariam M, Hagos S, et al. Seroprevalence of transfusion-transmissible infections among blood donors at National Blood Transfusion Service, Eritrea: a seven-year retrospective study. BMC infectious diseases. 2018;18(1):264. 
22.Nada H, Atwa M. Seroprevalence of HBV, HCV, HIV and syphilis markers among blood donors at Suez Canal University Hospital Blood Bank. J Blood Disord Transfus. 2013;5:177.

23.Stokx J, Gillet P, De Weggheleire A, Casas EC, Maendaenda R, Beulane AJ, et al. Seroprevalence of transfusion-transmissible infections and evaluation of the pre-donation screening performance at the Provincial Hospital of Tete, Mozambique. BMC infectious diseases. 2011;11(1):141.

24.Mavenyengwa RT, Mukesi M, Chipare I, Shoombe E. Prevalence of human immunodeficiency virus, syphilis, hepatitis B and C in blood donations in Namibia. BMC public health. 2014;14:424.

25.Biadgo B, Shiferaw E, Woldu B, Alene KA, Melku M. Transfusion-transmissible viral infections among blood donors at the North Gondar district blood bank, northwest Ethiopia: A three year retrospective study. PLOS ONE. 2017;12(7):e0180416.

26.Matee MI, Magesa PM, Lyamuya EF. Seroprevalence of human immunodeficiency virus, hepatitis B and $C$ viruses and syphilis infections among blood donors at the Muhimbili National Hospital in Dar es Salaam, Tanzania. BMC public health. 2006;6:21.

27.Deressa T, Birhan W, Enawgaw B, Abebe M, Baynes HW, Desta M, et al. Proportion and predictors of transfusion-transmissible infections among blood donors in North Shewa Zone, Central North Ethiopia. PLOS ONE. 2018;13(3):e0194083.

28.Noubiap JJ, Joko WY, Nansseu JR, Tene UG, Siaka C. Sero-epidemiology of human immunodeficiency virus, hepatitis $B$ and $C$ viruses, and syphilis infections among first-time blood donors in Edea, Cameroon. International journal of infectious diseases: IJID: official publication of the International Society for Infectious Diseases. 2013;17(10):e832-7.

29.Bazie E, Ali M, Hamza H, Magzoub O, Salih M, Haroun B. Sero-prevalence of viral transfusiontransmissible infections among blood donors at Kosti Teaching Hospital, White Nile State/Sudan. Int J Curr Microbiol App Sci. 2015;4(5):1132-8.

30.Bloch EM, Vermeulen M, Murphy E. Blood transfusion safety in Africa: a literature review of infectious disease and organizational challenges. Transfusion medicine reviews. 2012;26(2):164-80.

31.Bisetegen FS, Bekele FB, Ageru TA, Wada FW. Transfusion-Transmissible Infections among Voluntary Blood Donors at Wolaita Sodo University Teaching Referral Hospital, South Ethiopia. The Canadian journal of infectious diseases \& medical microbiology = Journal canadien des maladies infectieuses et de la microbiologie medicale. 2016;2016:8254343.

32.Stockdale AJ, Mitambo C, Everett D, Geretti AM, Gordon MA. Epidemiology of hepatitis B, C and D in Malawi: systematic review. BMC infectious diseases. 2018;18(1):516.

33.Nkrumah B, Owusu M, Averu P. Hepatitis B and C viral infections among blood donors. A retrospective study from a rural community of Ghana. BMC Res Notes. 2011;4:529. 
34.Mohammed Y, Bekele A. Seroprevalence of transfusion transmitted infection among blood donors at Jijiga blood bank, Eastern Ethiopia: retrospective 4 years study. BMC Res Notes. 2016;9:129.

35.Tenthani L, Haas AD, Egger M, Van Oosterhout JJ, Jahn A, Chimbwandira F, et al. Brief Report: HIV Testing Among Pregnant Women Who Attend Antenatal Care in Malawi. Journal of acquired immune deficiency syndromes (1999). 2015;69(5):610-4.

36.Tagny CT, Owusu-Ofori S, Mbanya D, Deneys V. The blood donor in sub-Saharan Africa: a review. Transfusion medicine (Oxford, England). 2010;20(1):1-10.

37.Song Y, Bian Y, Petzold M, Ung COL. Prevalence and trend of major transfusion-transmissible infections among blood donors in Western China, 2005 through 2010. PloS one. 2014;9(4):e94528.

38.The World Factbook [Internet]. 2019 [cited June 23, 2019]. Available from: https://www.cia.gov/library/publications/the-world-factbook/geos/mi.html.

39.Mkandawire-Valhmu L, Wendland C, Stevens PE, Kako PM, Dressel A, Kibicho J. Marriage as a risk factor for HIV: Learning from the experiences of HIV-infected women in Malawi. Global Public Health. 2013;8(2):187-201.

40.NSO. Malawi Demographic and Health Survey 2015-16. In: National Statistical Office (NSO) Malawi and The DHS Program ICF R, Maryland, USA, editor. 2017.

41.Biceroglu SU, Turhan A, Doskaya AD, Yamazhan T, Donmez A, Altuglu I, et al. Probable hepatitis C virus transmission from a seronegative blood donor via cellular blood products. Blood transfusion = Trasfusione del sangue. 2014;12 Suppl 1:s69-70.

\section{Figures}




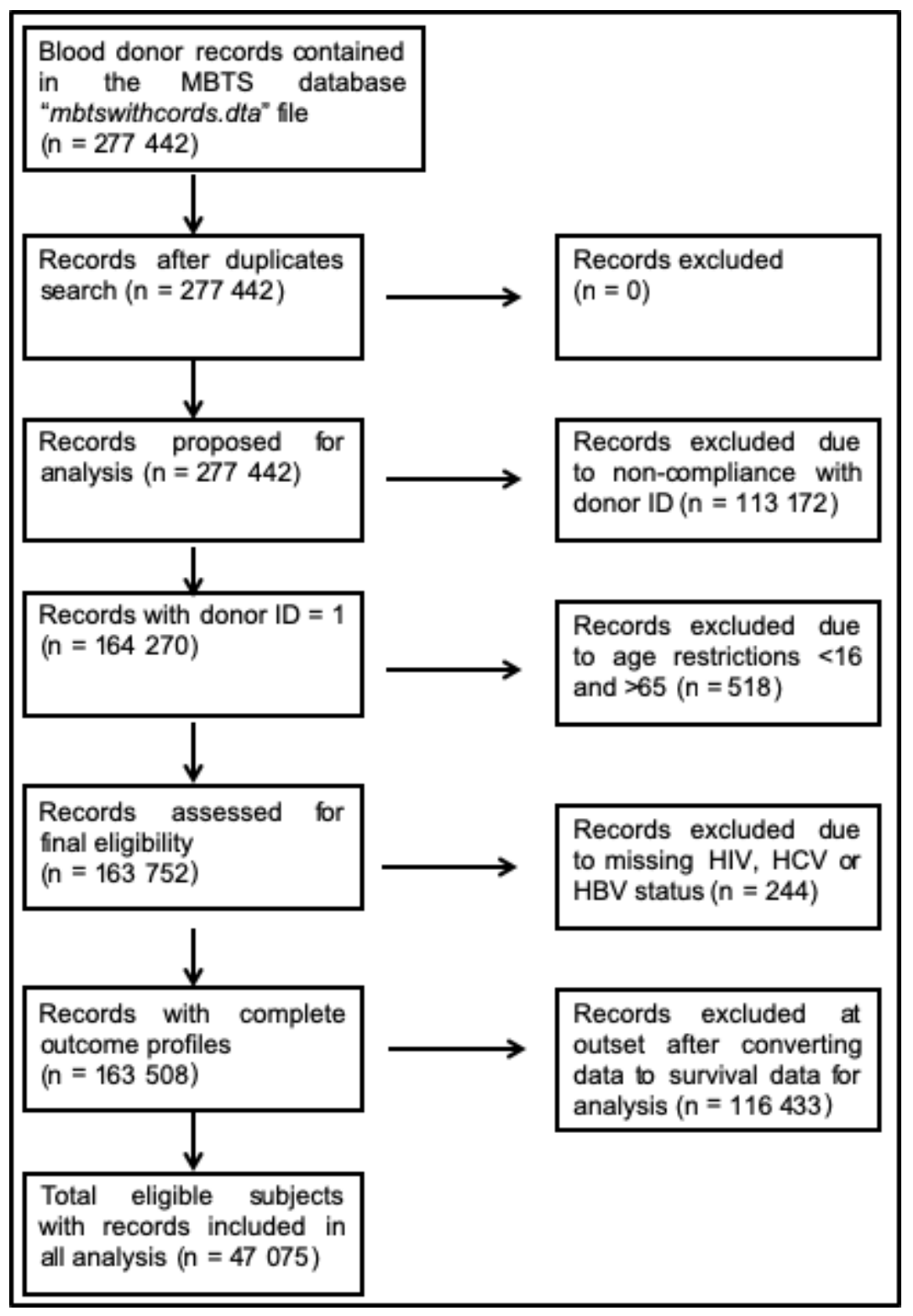

\section{Figure 1}

Flow chart diagram of blood donors enrolled in the study 

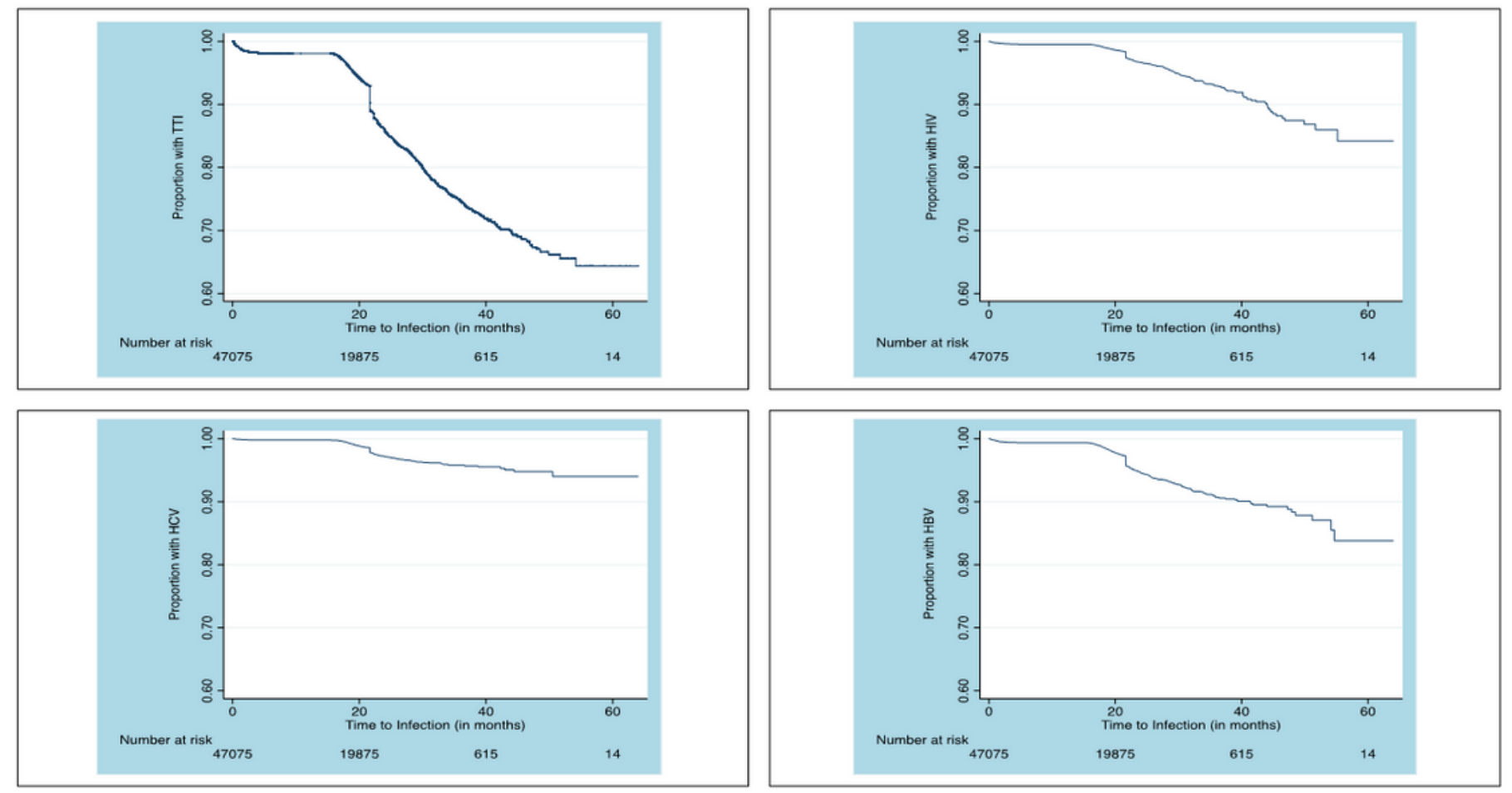

\section{Figure 2}

Overall Kaplan-Meier survival estimates of TTI, HIV, HCV and HBV $(\mathrm{N}=47,075)$
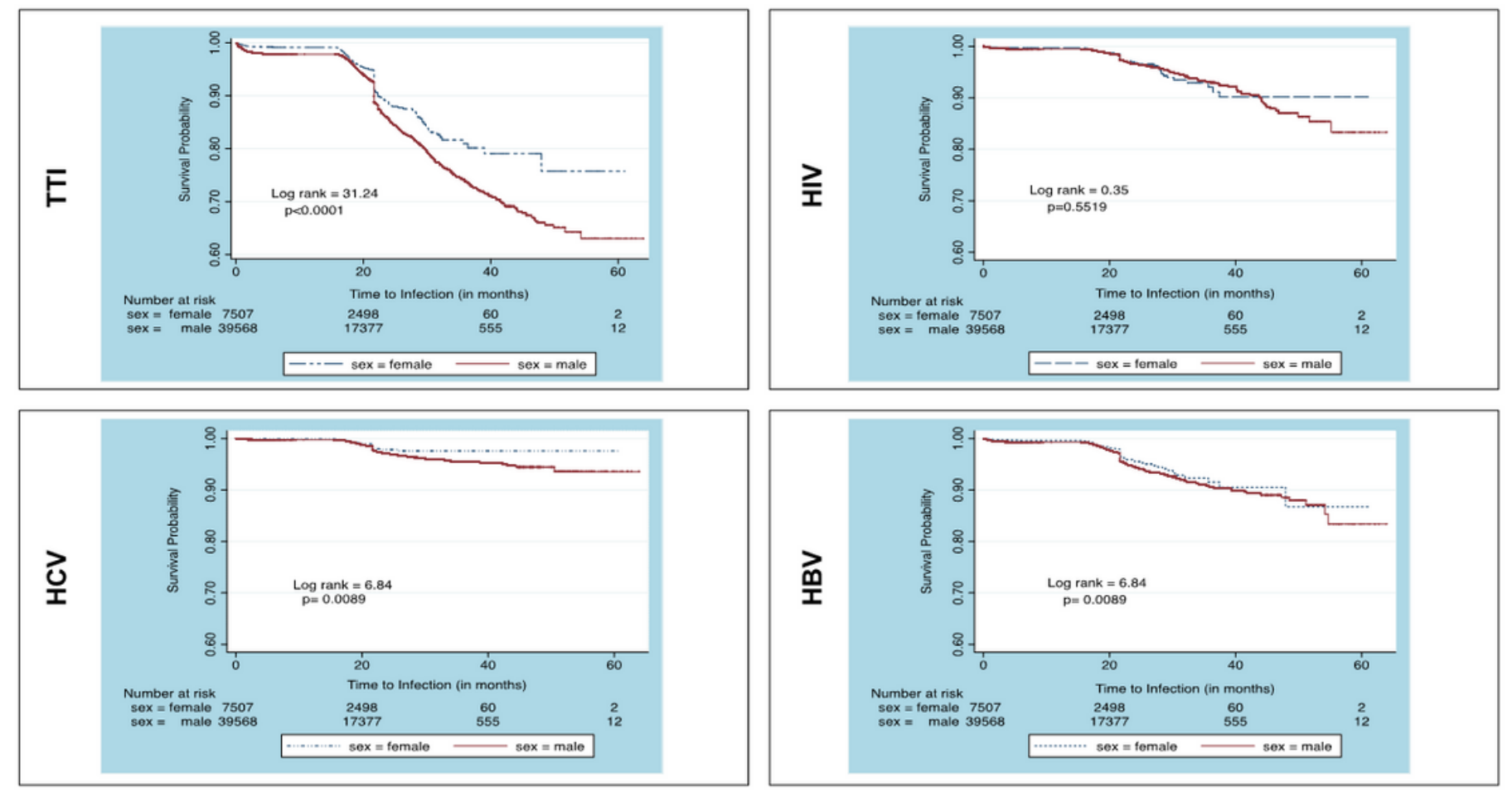
Figure 3

Kaplan-Meier survival estimates of TTI, HIV, HCV and HBV, by sex $(\mathrm{N}=47,075)$
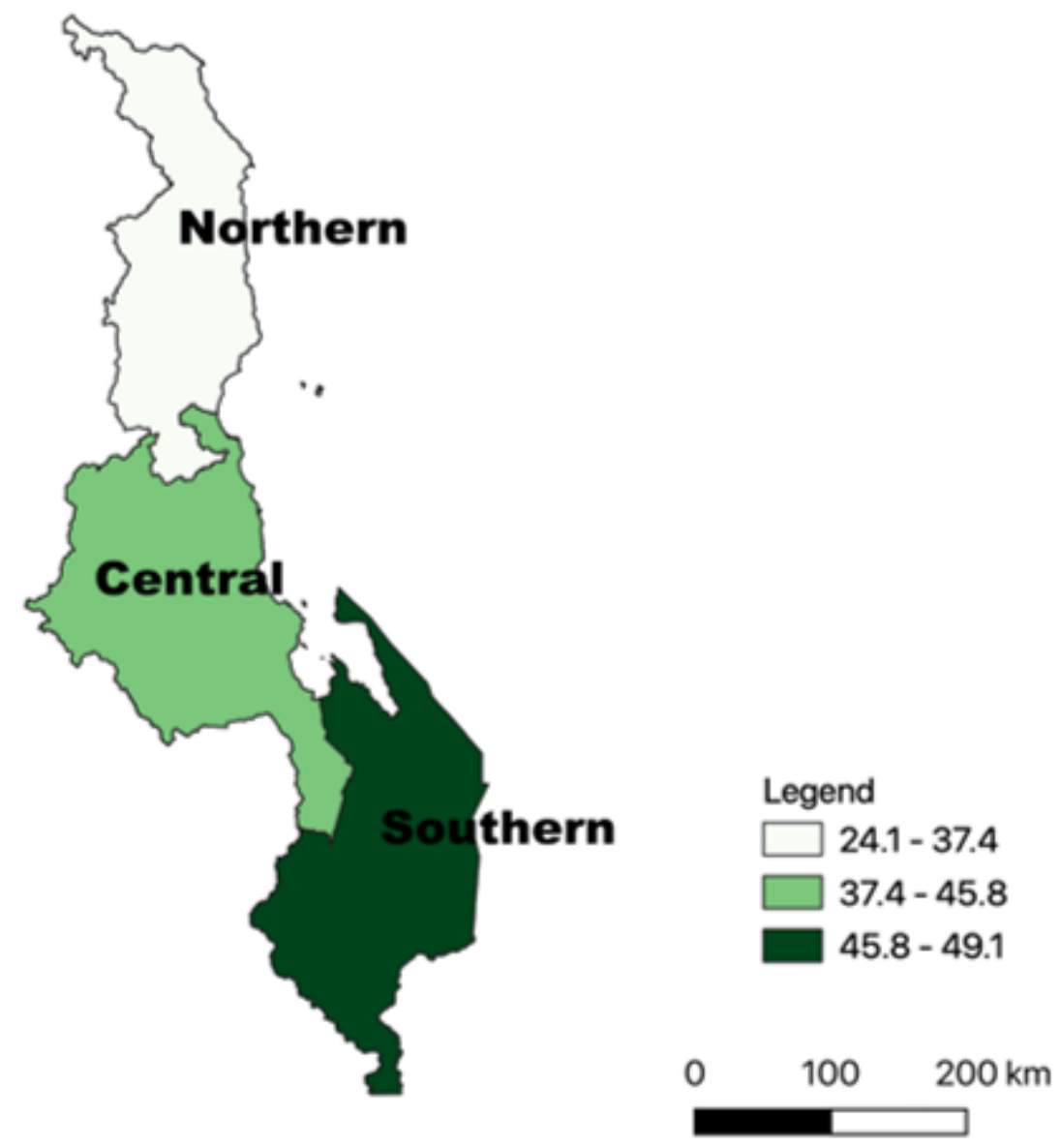

Figure 4

TTI incidence distribution in Malawian blood donors who donated blood from 2005-2015 